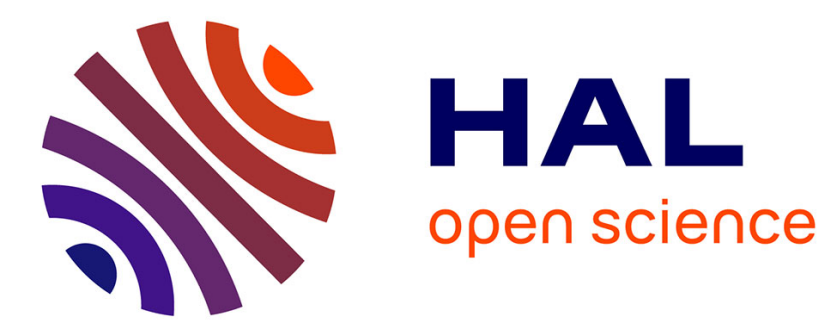

\title{
Biomechanical Assessment of the Individual Risk of Rupture of Cerebral Aneurysms: A Proof of Concept
}

Mathieu Sanchez, Dominique Ambard, Vincent Costalat, Simon Mendez, Franck Jourdan, Franck Nicoud

\section{- To cite this version:}

Mathieu Sanchez, Dominique Ambard, Vincent Costalat, Simon Mendez, Franck Jourdan, et al.. Biomechanical Assessment of the Individual Risk of Rupture of Cerebral Aneurysms: A Proof of Concept. Annals of Biomedical Engineering, 2012, 41 (1), pp.28-40. 10.1007/s10439-012-0632-2 . hal-00737354

\section{HAL Id: hal-00737354 \\ https://hal.science/hal-00737354}

Submitted on 1 Oct 2012

HAL is a multi-disciplinary open access archive for the deposit and dissemination of scientific research documents, whether they are published or not. The documents may come from teaching and research institutions in France or abroad, or from public or private research centers.
L'archive ouverte pluridisciplinaire HAL, est destinée au dépôt et à la diffusion de documents scientifiques de niveau recherche, publiés ou non, émanant des établissements d'enseignement et de recherche français ou étrangers, des laboratoires publics ou privés. 


\title{
Biomechanical assessment of the individual risk of rupture of cerebral aneurysms: a proof of concept
}

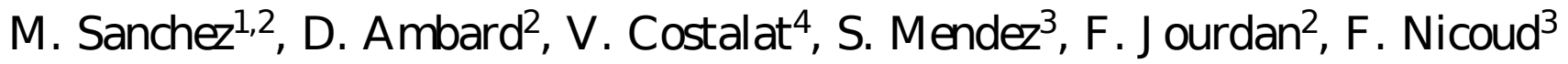 \\ J une 28, 2012 \\ ${ }^{1}$ Philips Healthcare, 33 rue de Verdun 92150 Suresnes cedex - France \\ ${ }^{2}$ Laboratoire de Mécanique et Génie Civil (LMGC), Université Montpellier 2, CNRS \\ UMR 5508, 34095 Montpellier Cedex 5, France \\ 3Institut de Mathématiques et de Modélisation de Montpellier (I3M), Université \\ Montpellier 2, CNRS UMR 5149, 34095 Montpellier cedex 5 - France \\ ${ }^{4} \mathrm{CHU}$ Gui de Chauliac, Service de neuroradiologie , 80 avenue Augustin Fliche 34295 \\ Montpellier Cedex 5
}




\begin{abstract}
This study is a step towards a new biomechanical-based measurement of the patient specific risk of rupture of cerebral aneurysms. Following a previous experimental investigation suggesting a correlation between the risk of rupture and the material properties of cerebral aneurysms, fluid-structure interaction simulations are performed to compare the deformations of a patient-specific aneurysm when using degraded or undegraded materials. Results show that material properties have a major impact on the magnitude of systolic/diastolic aneurysmal volume variations along the cardiac cycle. Changes in terms of aneurysmal volume variations depending on the tissue characteristics are shown to be measurable by medical imaging. A one-at-a-time data uncertainty analysis is also presented and shows the robustness of this result to input data uncertainties. The study thus suggests that aneurysmal volume variations may be used as the basis of a biomechanical index of rupture risk.
\end{abstract}

Keywords: FSI, cerebral aneurysm, hyperelasticity, rupture risk assessment 


\section{Introduction}

The prevalence of unruptured intracranial aneurysms in the general population, as reported by Wardlaw et al. [36] ranges between $3 \%$ and 6.6\%. The incidence of ruptured aneurysms is however low, with approximately 10 cases in every 100,000 people per year, which suggests that very few aneurysms break. Subarachnoid hemorrhage is the consequence of aneurysm rupture and approximately $12 \%$ of patients die before receiving medical attention, $40 \%$ of hospitalized patients die within one month after the event, and more than one third of those who survive have major neurological deficits. In contrast, endovascular treatment of unruptured aneurysm is safe with a $0.03 \%$ mortality rate. Unruptured intracranial aneurysms may be diagnosed clinically in a setting of subarachnoid hemorrhage caused by the rupture of another existing aneurysm, during screening in a setting of positive family history of ruptured intracranial aneurysm or autosomal dominant polycystic kidney disease, in the investigation of clinical symptoms relative to cranial nerve compression or hydrocephalus related to the aneurysm, or may be diagnosed fortuitously during routine Computed Tomography scanner (CT scan) or Magnetic Resonance Imaging (MRI) investigations for problems unrelated to the aneurysm. Whatever the case, unruptured intracranial aneurysms represent a dilemma for physicians, who have to evaluate the risk of aneurysm rupture with respect to its natural history against the risk of morbidity and mortality from an endovascular or surgical repair. With CT scan and MRI being more frequently and widely used, a growing number of intracranial aneurysms are being diagnosed, posing the problem of which aneurysms harbor a sufficiently high risk of rupture to merit endovascular or surgical repair.

Recent publications have addressed this issue and have demonstrated that, among other variables affecting the natural history of aneurysms, size and location represent independent predictors of both risk of rupture and surgical/endovascular repair outcomes $[14,21]$. Other parameters, such as irregular aneurysm shape and the presence of blebs are recognized as 
translating an area of weak wall structure and high risk of rupture. Rapid aneurysm growth is also likely a risk factor for rupture[18].

From a mechanical point of view, the rupture of an aneurysm occurs when wall tension exceeds the strength of the wall tissue. Since these quantities cannot be assessed from medical imaging, a natural approach is to compute the wall tension and set a rupture threshold. Recently, it has been showed that numerical simulations based on patientspecific image data may be used to simulate wall shear stress (WSS) and pressure in the circle of Willis [24]. Others have used computations to analyze the flow in cerebral aneurysms, with focus on WSS, which is also thought to be associated with aneurysm formation and rupture $[6,7,13]$. Some studies consider the coupled fluid - structure interaction problem (FSI) where the flow equations for blood are solved together with the structural equations for the tissue [8]. Because mechanical properties for cerebral arteries and aneurysms are very scarce most of the studies based on a FSI [4, 22, 25, 30, 35] framework do not use experimental mechanical behavior of the aneurysm wall as input. A few exceptions exist for the case of the abdominal aorta aneurysms $[33,34]$ but not for the intracranial aneurysms.

In the study of Costalat and al [9], the aneurysm wall properties were characterized and a classification of the aneurysm wall behavior was made. The main output of this study is that the medical status of the aneurysm (undegraded, degraded, close to rupture or ruptured) is strongly correlated with the aneurysmal tissue property (stiff, intermediate, soft). A rupture criterion could be directly evaluated by assessing the state of the material and using an inverse method. By combining medical imaging and FSI computations, it should be possible to determine the mechanical property of the aneurysm wall. This approach can be successful only if a significant difference of the volume variation over the cardiac cycle is observable for two different materials (soft and stiff) with medical imaging. For example, Karmonik et al, in their study [16], could measure a displacement as small as $0.04 \mathrm{~mm}$ with a $1.5 \mathrm{~T}$ MRI. 


\section{Materials and Methods}

\subsection{Identification of mechanical behavior of aneurysm wall}

The details of the study establishing the correlation between the aneurysm status and tissue mechanical properties are given in [9]; the methodology and main results are briefly given in the following for completeness. The same kind of study has been done by Duprey and al. [11] for the thoracic aortic aneurysm.

Samples were extracted from surgically removed aneurysm sacs coming from several University Hospitals. In order to conserve the mechanical properties of the aneurysm wall, a specific conservation protocol was applied in each center by means of a dedicated histopathological removal kit available in the neurosurgical operating room. The resected aneurysm was initially inserted in a tube containing a Ringer lactate, $10 \%$ DMSO solution. This first tube was then placed in a larger tube containing isopropanol. This combination of the two tubes was placed in a freezer $\left(-80^{\circ} \mathrm{C}\right)$.

Each sample was then defrozen and studied as appropriate for biomaterials $[3,15,17$, 29]: One hour before mechanical testing, aneurysms sample were thawed at ambient temperature. Under microscopy, the aneurysmal wall samples were dissected in a meridional manner in order to obtain a regular rectangular piece. Only the meridional axis of the aneurysm was chosen in order to preserve maximum length of the aneurysmal tissue in the sample, given the very small size of each specimen and the fragility of the tissue. The aneurysm strips were physically measured and then glued on each extremity to aluminum grips. Meanwhile, physiological isotonic liquid was warmed to $40{ }^{\circ} \mathrm{C}$ inside the traction test machine. A uniaxial stretch test was carried out on the sample within the warmed physiological liquid in order to simulate the in vivo conditions. This testing device was composed of a Texture Analyzer (TA-XT2, Stable Microsystems, UK) with a 50 N load cell and an optical microscope (ZEISS) equipped with a digital video camera . The uniaxial stretch test consisted of a sequence of $10 \%$ length displacement of the sample, in 5 repeated 
cycles, while registering the traction force applied. In accordance to standard mechanical testing protocol for biological tissue, the specimens were first preconditioned during the first four cycles. The extension rate was $0.01 \mathrm{~mm} / \mathrm{s}$ and the tension load was recorded every $0.01 \mathrm{~s}$. Velocity of the solicitations was small enough to neglect viscous phenomena. Strain/stress curves for each sample were generated (Fig. 1).

Using theses measurement series, a behavior model of the tissue was proposed for large displacements in order to represent the evolution of the stress in the materials [13]. For this purpose the assumption that the material is isotropic and incompressible was made and the hyperelastic model of Mooney-Rivlin with 3 parameters was selected (1). It reads:

$$
W=C_{10}\left(I_{1}-3\right)+C_{01}\left(I_{2}-3\right)+C_{11}\left(I_{1}-3\right)\left(I_{2}-3\right),
$$

where $W$ is strain energy potential, $I_{1}=\operatorname{tr}(\mathbf{C})$ and $I_{2}=\frac{1}{2}\left(\operatorname{tr}^{2}(\mathbf{C})-\operatorname{tr}\left(\mathbf{C}^{2}\right)\right)$ are the first and second strain invariants of the right Cauchy-Green deformation tensor $\mathbf{C}$ and $C_{10}, C_{01}$, $C_{11}$ are the material coefficients. The latter were tuned in order to mimic the mechanical behavior of each sample. The 1D curve from the experimentation is used to determine the coefficient thanks to an identification process. In absence of relevant data regarding the material anisotropy and inhomogeneity, these coefficients were then used without any change in the 3D version of the model (1). The experimental study demonstrated three main tissues classes: soft, stiff and intermediate. $C_{11}(1)$ is the material coefficient the most representative of the behavior of the aneurysm wall.

All unruptured aneurysms presented a stiffer tissue than the ruptured aneurysms (Fig. 1). Their mechanical behavior is either stiff or intermediate. Conversely, all the ruptured aneurysms correspond to a soft tissue. In order to further analyze whether the difference in tissue behavior leads to measurable differences in aneurysm variation over the cardiac cycle, two aneurysms representative of the average of the stiff (undegraded) and the soft (close to the rupture) classes were selected for the FSI computations described in the next section. The corresponding parameters are given in Table 1 while the strain/stress curves 
are displayed in Fig. 1 


\section{$2.2 \quad$ Numerical Method}

In the following, the mechanical behaviors assessed experimentally from actual aneurysm samples are used to feed FSI computations of a realistic aneurysm geometry. The physical and numerical characteristics of the computations are now presented.

\subsubsection{D object}

The geometry is obtained from images gained from an MRI of a specific patient exam. The first step is to create a 3D object from 2D images. The software ScanIP is used for this purpose [39]. The next step is to clean up the geometry in keeping only the parent artery and then to reduce the region of interest by cropping. At last, a low-pass fitter is applied to the images to reduce the noise-to-signal ratio and then the result is then exported as an STL file (Fig. 2). The neck diameter is $N=6.25 \mathrm{~mm}$ and the dome length is $D=7.6$ $\mathrm{mm}$, which gives a ratio $\frac{D}{N}=1.216$. The parent artery diameter is $d=3.3 \mathrm{~mm}$.

\subsubsection{Physical modeling}

The blood flow within the computational domain is described by the incompressible NavierStokes equations (2), (3).

$$
\begin{aligned}
& \rho_{f} \frac{d \overrightarrow{v_{f}}}{d t}=\overrightarrow{\operatorname{div}}\left(\sigma^{f}\right), \\
& \operatorname{div}\left(\overrightarrow{v_{f}}\right)=0,
\end{aligned}
$$

where $\overrightarrow{v_{f}}$ is the time dependent flow velocity vector, $\rho_{f}$ is the mass density of blood and $\sigma^{f}$ is the strain for the fluid part. The flow is assumed to be laminar, a reasonable assumption given the moderate value of the Reynolds number $(R e \simeq 300)$, defined as:

$$
R e=\frac{\rho_{f} v_{f} d}{\mu},
$$

where $v_{f}$ is the bulk velocity of the blood $\left(v_{f} \simeq 0.4 \mathrm{~m} \cdot \mathrm{s}^{-1}\right), d$ is the diameter of the parent artery $(d=3.3 \mathrm{~mm})$ and $\mu$ is the dynamic viscosity of the blood. Blood is 
modeled as a Newtonian fluid (5), of density of $\rho=1050 \mathrm{~kg} / \mathrm{m}^{3}$ and dynamic viscosity of $\mu=0.004$ Pa.s $[1,30,37,38]$.

$$
\sigma^{f}=-p_{f} \mathbf{I}+2 \mu \mathbf{D}
$$

where $p_{f}$ is the pressure and $\mathbf{D}$ is the rate of deformation tensor. More sophiscated models can be used in order to account for non-Newtonian effects, especially in the aneurysmal sac (see for instance Cebral et al. [5] and Sforza et al. [26]). However, these effects are expected to be prevalent only when dealing with local quantities like wall shear stress. This is not the case in the present paper where the aneurysmal volume, which is primarily influenced by the inviscid pressure forces, is analyzed.

Two different materials [37] are used for the structural parts, one for the aneurysmal sac (hyperelastic) and one for the artery (linear elastic) to focus the attention on the aneurysmal sac and to reduce the computational time. The structure equation solved for the artery and aneurysmal sac reads (6):

$$
\rho_{s} \frac{\partial \overrightarrow{v^{s}}}{\partial t}=\overrightarrow{\operatorname{div}}\left(\sigma^{s}\right)
$$

where $\sigma^{s}$ is the Cauchy strain for the structural part.

The aneurysm wall is modeled as an isotropic incompressible hyperelastic material (7):

$$
\sigma^{s}=\rho_{s} \mathbf{F}\left(\frac{\partial W}{\partial \mathbf{E}}\right) \mathbf{F}^{t}-p_{s} \mathbf{I}
$$

where $\mathbf{F}$ is the deformation gradiant tensor and $\mathbf{E}$ is the Green Lagrangian strain tensor. The material used for the parent artery has the following properties: Young modulus $E=$ $3 \mathrm{MPa}$, Poisson ratio $\nu=0.49$, specific mass $\rho_{s}=2300 \mathrm{~kg} / \mathrm{m}^{3}$ and thickness $e_{p a}=0.6 \mathrm{~mm}$ $[31,32]$. The thickness of the aneurysmal wall is $e=0.38 \mathrm{~mm}$.

\subsubsection{Boundary conditions}

For the FSI analysis, a normal pressure to the external surface, due to the intracranial pressure, of $2000 \mathrm{~Pa}$ is imposed to the wall [28]. Realistic hemodynamic conditions are 
imposed as obtained from a specific patient examination by using MRI in phase contrast. More precisely, the inlet volumetric blood flow rate and the pressure at the artery outlet imposed during the calculation are shown in Fig. 3.

The flow boundary conditions and the morphological data were not measured on the same patient as the geometry: such a complete dataset for a unique patient was not available. However, hemodynamic boundary conditions are relevant to actual physiological conditions and good enough for the proof of concept we attend to demonstrate in the present study.

\subsubsection{FSI computations}

The structural mesh contains 3,110 shell elements (in order to decrease the computational time and because of the small thickness of the aneurysm wall) and the fluid mesh is composed of 102,562 tetrahedral elements (Fig. 4). For each FSI computation, a time step of $0.004 \mathrm{~s}$ was used.

Computations are performed using the software Ansys V.13. The fluid motion equations are solved with the software CFX, which uses the finite volume approach and the NewtonRaphson method for solving the subsequent non linear system. For the structural part, Ansys employs the finite element method and the Newton-Raphson algorithm. The wall pressure resulting from the fluid is imported as boundary conditions in the structural analysis; this procedure is carried out in an iterative manner within each time step. It is an iterative implicit coupling. At the interface of the two physical domains, the element type differs and the nodes of the two meshes do not coincide. In order to solve this interfacial problem, surface interpolation is carried out [2].

\subsubsection{Initial stress}

To perform a finite element computation with an hyperelastic behavior law as described in section 2.1, the knowledge of the stress-free and no strain configuration, not accessible 

using computations the stress-free state of the geometry. We put forward an alternative approach, which consists in using a representative configuration, for which we know the geometry and the pressure conditions. This configuration is given by medical imaging synchronized with the cardiac cycle and will be referred to as the geometry at acquisition. Note that 3D rotational angiography (3DRA) produced only an average geometry over the cardiac cycle. However, since $80 \%$ of the cardiac cycle corresponds to the diastolic pressure, we assumed that the geometry and the pressure are closed to the diastolic state (Line $\mathrm{C}$ in Fig. 3). In a close future the appropriate MRI sequence (4D phase contrast MRI) with cardiac gating will be used to obtain geometry and a pressure at a particular time of the cardiac cycle. For this geometry, under the estimated pressure (line $\mathrm{C}$ in Fig. 3, $\left.p_{\text {ext }} \simeq 9000 \mathrm{~Pa}\right)$, one needs to determine the stress in each Gauss point of the structural mesh. The latter is assessed by assuming that the inertial effects are negligible in the wall motion. Then a computation is made for a linear elastic material under small strain assumption to solve the equilibrium equation.

$$
\overrightarrow{\operatorname{div}}\left(\sigma^{s}\right)=\overrightarrow{0}
$$

where $\sigma^{s}$ is the strain tensor for the structural part. Note that the stress results of this equation does not bring into play the material behavior characteristics. Note also that neglecting the inertial effects was validated by comparing two FSI computations, one with $\rho_{s}=2300 \mathrm{~kg} / \mathrm{m}^{3}$ for the tissue, the other one with $\rho_{s}=0$, and checking that the results were very similar (not shown). The stress field obtained from Eq (8) is thus used as the initial condition for all the FSI computations. Using this representative stress, from now on, Ansys is able to compute the stress and strain states at any time over the cardiac cycle. This technique is valid only if the inertial effects can be neglected for this assessment;

In Fig. 5, a summary of a representation on the stress/strain graph of the different conditions is proposed. 
- State 0 corresponds to the aneurysmal sac being cut off; in this case, no initial stress is present and $S=0$.

- State 1 corresponds to the diastolic condition with the nominal stress associated $S_{d}$. (Point A in Fig. 3 and Fig. 5)

- State 2 corresponds to the systolic condition with the nominal stress associated $S_{s}$. (Point B in Fig. 3 and Fig. 5)

- State 3 corresponds to the geometrical acquisition condition with the nominal stress associated $S_{a}$. (line C in Fig. 3 and point C in Fig. 5) 


\section{Results and parametric study}

\subsection{Representative computation}

The aim is to determine if significant geometrical differences measurable using medical imaging exist between a soft (close to the rupture) aneurysm and a stiff (healthy) aneurysm - For this purpose, two representative FSI computations were performed for the stiff and soft tissues using the numerical procedure described in subsection 2.2.

Two quantities are observed and discussed in the following:

- $\mathrm{V}_{\text {diff }}$ : the volume variation of the aneurysm sac (lumen because of the incompressibility of the material model) over the cardiac cycle.

- $\mathrm{D}_{\max }$ : the maximal mesh displacement on the aneurysm sac.

$\mathrm{V}_{\text {diff }}$ represents a global information about the aneurysmal sac, contrary to $\mathrm{D}_{\max }$ which is the maximal displacement of a point of the mesh and gives a local information. Both quantities can be easily measured by MRI

From table 2, significant differences are observable between the soft and stiff aneurysm (Fig. 6). The relative difference between the two maximal mesh displacements is about $200 \%$ and $500 \%$ for the volume variation. The variation of the volume of the aneurysm is roughly 10 times larger in the case of the soft tissue. The number of elements of the meshes and the time step were determined to obtain the best compromise between result accuracy and CPU time. Other simulations were made with a time step of $0.0004 \mathrm{~s}$ (ten times smaller than the representative simulations) and meshes 10 times finer (about 30,000 elements for structural side and 1,000,000 elements for the fluid side). Despite a significant increase of the CPU time, the results were similar; for example for the soft case, the maximal mesh displacement is $0.78 \mathrm{~mm}$ (difference of $1.3 \%$ ) an the volume variation is $44.3 \mathrm{~mm}^{3}$ (difference of $0.7 \%$ ).

Such a difference is most probably measurable by modern medical imaging. This information could then be used to assess indirectly the status of the tissue (soft/stiff, that is to 
say close to the rupture/undegraded). Note however that a common difficulty when computing blood flows under in vivo conditions is the uncertainty on the input data. To this purpose, a parametric study was performed and is described in the following sub-sections, in order to make sure uncertain quantity will be considered separately and results will be compared to the representative computations presented in this section. That to make sure that the computed difference between the evolution of soft and stiff aneurysm is robust to uncertainties in input data.

\subsection{Influence of the initial stress}

Assessing the initial stress for conducting proper FSI computation is a difficult task, which makes input data quite uncertain. The uncertainty on $S_{a}$ comes notably from the limit of the precision of the registration of the blood pressure during medical imaging. Starting from the initial stress $\left(S_{a}\right)$ assessed as described in section 2.2.4, two types of FSI simulations were performed to evaluate the influence of this parameter. The first one without initial stress $(S=0)$ and the second one with an initial stress multiplied by two $\left(S=2 S_{a}\right)$. The main results are gathered in table 3 and 4 and (Fig. 7).

\subsection{Influence of the flow boundary conditions}

Because they are difficult to measure from medical imaging, boundary conditions are also quite uncertain when performing FSI computation under in vivo conditions.

\subsubsection{Influence of a simultaneous variation of the inlet and the outlet bound- ary conditions}

The imposed flow at the inlet and the imposed pressure at the outlet were modified in this section. Two FSI computations were performed for the stiff and soft aneurysms. In the first case, an increase of $10 \%(\mathrm{CL}+10 \%)$ of the flow boundary conditions (inlet volumetric flow 
rate and outlet pressure) is imposed and in the second one, a decrease of 10\% (CL-10\%) is imposed.

Results are gathered in table 3 and 4 and Fig. 7.

These results suggest that the change of the boundary flow conditions $( \pm 10 \%)$ generates a limited impact on the volume variation of the aneurysm sac over the cardiac cycle in both stiff and soft cases (respectively $\pm 7.5 \%$ and $\pm 5 \%$ ). Their influence is a little bit more significant locally, as shown by the maximal mesh displacement results, for both stiff and soft aneurysms (respectively $\pm 14 \%$ and $\pm 9 \%$ ). This impact is in any case small enough to still distinguish soft and stiff aneurysmal sacs.

\subsubsection{Influence of the volumetric flow rate}

In this section, only the volumetric flow rate (VFR)at the inlet was modified. An increase and a decrease of $30 \%$ (VFR $+30 \%$ and VFR -30\%) were applied on both stiff and soft materials.

Results are gathered in table 3 and 4 and Fig. 7.

The variation of the volumetric flow rate have an impact on $\mathrm{D}_{\max }( \pm 16 \%$ for the soft and $\pm 30 \%$ for the stiff $)$ and $V_{\text {diff }}( \pm 18 \%$ for the soft and $\pm 24 \%$ for the stiff $)$. But, despite an important increase and decrease of the volumetric flow rate, a significant difference is still observable between the stiff and the soft aneurysm.

\subsubsection{Influence of the outlet pressure}

To quantify the influence of the outlet pressure, only the difference between the diastolic pressure and the systolic pressure $(\triangle \mathrm{P})$ were modify. Two cases were performed, for $\triangle \mathrm{P}$ $=35 \mathrm{mmHg}$ and $\triangle \mathrm{P}=45 \mathrm{mmHg}$.

Results are gathered in table 3 and 4 and Fig. 7.

Whatever the values of $\triangle \mathrm{P}$, the results showed that a significant difference is observable between the stiff and the soft materials. 


\subsection{Influence of the parent artery properties}

To measure the influence of the parent artery properties, the Young modulus of the artery $\left(E_{a}\right)$ was modified, during the FSI computations, first to $1.5 \mathrm{MPa}$ and then to $6 \mathrm{MPa}$ instead of $E_{a}=3 \mathrm{MPa}$ in the representative computations.

Results are gathered in table 3 and 4 and Fig. 7.

A significant difference is still observable between a wall composed of the stiff material with the softer parent artery properties and a wall composed of the soft material with the stiffer parent artery properties.

Overall, the influence of the parent artery properties can almost be neglected given the results of the volume variation in both stiff and soft cases. The maximal mesh displacement gives a local information, contrary to the volume variation which gives a global information. The variation of the maximal mesh displacement measured by the software depends strongly of the displacement of the artery (notably for $E=1.5 \mathrm{MPa}$ ). The deformation of the artery induces an additional displacement of the mesh of the aneurysmal sac. Then, the bulge of the artery is added to the displacement of the sac in relation to the artery to obtain the total maximal mesh displacement $\mathrm{D}_{\max }$. $\mathrm{V}_{\text {diff }}$ is a more relevant quantity than $\mathrm{D}_{\max }$ to assess the material state of the aneurysmal sac.

\subsection{Influence of the intracranial pressure}

The intracranial pressure is difficult to measure and the influence of the error on its value is another uncertainty to quantify. Two cases were considered on both stiff and soft aneurysms. In the first case, no intracranial pressure was applied $\left(P_{e x t}=0 \mathrm{~Pa}\right)$. In the second one, a pressure of $5000 \mathrm{~Pa}$ was applied $\left(P_{\text {ext }}=5000 \mathrm{~Pa}\right)$ instead of $P_{\text {ext }}=2000 \mathrm{~Pa}$ in the representative computation [28]. This increase of the external pressure corresponds to pathologies where the intracranial pressure can reach a value between 30 and $35 \mathrm{mmHg}$ and becomes dangerous for the patient. 
The variation of the intracranial pressure has almost no influence on the maximal mesh displacement and on the volume variation in both, the stiff and the soft, cases. That is a consequence of the computation of the initial stress. The computation of the initial stress is made by taking into consideration the external pressure and the pressure at the acquisition time. The variation of the external pressure modifies the state of the initial stress. The structure is in equilibrium under this load; the deformation of the wall results only from the variation of the pressure between the diastolic and systolic state (Fig. 5).

\subsection{Influence of the thickness of the aneurysmal wall}

The thickness of the wall is difficult to evaluate from actual medical imaging. That is why we need to know the impact of an error on this parameter. For this purpose, two extreme thicknesses $(\mathrm{e}=0.2 \mathrm{~mm}$ and $\mathrm{e}=0.6 \mathrm{~mm}$ ) were tested in our FSI computations.

Results are gathered in table 3 and 4 and in Fig. 7.

As expected, the thickness of the aneurysmal wall has an important influence on the results of the FSI, especially on the volume variation for both stiff and soft aneurysm. Note that the difference is still important (far from a cross-road) between the soft material with the thickest wall and the stiff material with the thin wall.

The thickness of the aneurysm wall can be measured by a black blood MRI sequence [23], which gives access to a homogenous thickness of the wall not so far from the real one. Still, wall thickness is not known precisely. However, the presented results show that whatever the thickness, a soft aneurysm deforms more than a stiff one. 


\section{Discussion}

Recent experimental measurements on real intracranial artery tissues extracted from aneurysm during surgery suggest a strong correlation between the mechanical properties of the material and the risk of rupture of aneurysms [9]. The present study demonstrates that, on a particular case of saccular aneurysm.

From the FSI computation with initial stress, there is a significant difference between the displacements and volume variations corresponding to the soft and stiff tissues. This results are coherent with a recent clinical study by Hayakawa et al [12], which shows a difference of the pulsation of the aneurysm sac (or volume variation) between ruptured aneurysms and unruptured aneurysms. They came to the conclusion that the detection of pulsation in an unruptured aneurysm is therefore a clue of an important risk of rupture of the aneurysm. Note also that the difference observed in our FSI simulation are large enough to be detected by advanced MRI protocole since displacements as small as 0.04 $\mathrm{mm}$ are measured recently by Karmonik et al [16].

More importantly, this result is robust regarding the numerous sources of uncertainty involved in such FSI computations. Numerous parameters are not known precisely when computing the FSI problem for a specific patient: wall thickness, fluid boundary conditions, artery properties, intracranial pressure,etc. Thanks to a parametric study, the present paper demonstrates that such uncertainties do change the details of the results, but not the main conclusion: whatever the values of the uncertain parameters (in a range of acceptable variations), the soft aneurysm deforms more than the stiff one.

A patient specific risk of rupture could be based on the comparison of the volume variation $\left(\mathrm{V}_{\text {diff }}\right)$ of the aneursymal sac of the FSI computations (for the stiff and the soft materials) and the observed $\mathrm{V}_{\text {diff }}$ of the patient (Fig. 8). For example, if the $\mathrm{V}_{\text {diff }}$ value measured on a patient aneurysm is close to $\mathrm{V}_{\text {diff }}$ value obtained in the simulation using the soft material, then the aneurysm is close to the rupture according to the classification 
of Costalat et al.

A limitation of this study is to not introduce the aneurysmal environment in the FSI computations. This environment would probably lead to important changes in the geometrical variations of the aneurysm continent on the localization of this one (friction and contact of the wall with other structures, suppression of degrees of freedom...). The assumption we made about the wall is another limitation. In the present study, the aneurysm wall is considered as isotropic and homogenous but the anisotropy of this kind of biological material is known. However, due to the smallness of the samples, biaxial testing to characterize the anisotropy of the wall could not be realized. Isotropy and homogeneity were thus assumed because they most probably do not change the trends observed when comparing soft and stiff materials. Note that the methodology presented in this paper could be readily extended to more complex rheologies as soon as biaxial testing are made available Another limitation comes from the fact that the data used to feed the FSI computations do not come from a single patient. Note however that these data are all realistic and that their collection on a single patient would be feasible in the medical routine. We thus believe that the results presented in this paper demonstrate the possibility to build a biomechanical based criterion for the patient specific rupture risk assessment. The fact that the uncertainties were evaluated only one by one for each parameter is another limitation. The evaluation of the influence of combined parameter uncertainties is another step to perform.

Obviously, the generality of such a conclusion has to be demonstrated in further studies, but given the correlation between volume variation, observable from MRI, and the aneurysm status observed in [9], the present paper represents the first step of a proof of concept in the evaluation of an individual risk of rupture of intracranial aneurysms. 


\section{Acknowledgments}

The authors thank Philips Healthcare, Inc. for the financial support and Ansys, Inc. for their technical support. The authors are also grateful to Simpleware, Inc. for providing the software Scan IP and for their technical support. 


\section{References}

[1] L. Ai and K. Vafai. A coupling model for macromolecule transport in a stenosed arterial wall. International Journal of Heat and Mass Transfer, 49:1568 - 1591, 2006.

[2] M. Alnaes, J. Isaksen, K. Mardal, B. Romner, M. Morgan, and T. Ingebrigtsen. Computation of hemodynamics in the circle of Willis. Stroke, 38:2500 - 2505, 2007.

[3] D. Ambard and F. Cherblanc. Mechanical behavior of annulus fibrosus: a microstructural model of fibers reorientation. Annals of Biomedical Engineering, 37(11):2256$2265,2009$.

[4] S. Balocco, O. Camara, E. Vivas, T. Sola, L. Guimaraens, H. Gratama van Andel, C. Majoie, J. Pozo, B. Bijnens, and A. Frangi. Feasibility of estimating regional mechanical properties of cerebral aneurysms in vivo. Medical Physics, 37(4):1689$1706,2010$.

[5] J. Cebral, M. Castro, C. Putman, D. Millan, and A. Frangi. Efficient pipeline for image-based patient-specific analysis of cerebral aneurysm hemodynamics: Technique and sensitivity. IEEE Transactions of Medical Imaging, 24(4):457 - 467, 2005.

[6] J. Cebral, F. Mut, J. Weir, and C. Putman. Association of hemodynamic characteristics and cerebral aneurysm rupture. American Journal of Neuroradiology, 32(10):264 $-270,2011$.

[7] J. Cebral, F. Mut, J. Weir, and C. Putman. Quantitative characterization of the hemodynamic environment in ruptured and unruptured brain aneurysms. American Journal of Neuroradiology, 32(10):145 - 151, 2011.

[8] S. Chung and K. Vafai. A coupling model for macromolecule transport in a stenosed arterial wall. Journal of Biomechanics, 45:371 - 381, 2012. 
[9] V. Costalat, M. Sanchez, D. Ambard, and al. Biomechanical wall properties of human intracranial aneurysms resected following surgical clipping. Journal of Biomechanics, 44(15):2685 - 2691, 2011.

[10] S. de Putter, B. Wolters, M. Rutten, M. Breeuwer, F. Gerritsen, and F. van de Vosse. Patient-specific initial wall stress in abdominal aortic aneurysms with a backward incremental method. Journal of Biomechanics, 40(5):1081 - 1090, 2007.

[11] A. Duprey, K. Khanafer, M. Schlicht, S. Avril, D. Williams, and R. Berguer. Ex vivo characterization of biomechanical behavior of ascending thoracic aortic aneurysm using uniaxial tensile testing. European Journal of Vascular and Endovascular Surgery, 39(6):700 - 707, 2009.

[12] M. Hayakawa, S. Maeda, A. Sadato, T. Tanaka, T. Kaito, N. Hattori, T. Ganaha, S. Moriya, K. Katada, K. Murayama, Y. Kato, and Y. Hirose. Detection of pulsation in ruptured and unruptured cerebral aneurysms by electrocardiographically gated 3-dimensional computed tomographic angiography with a 320- row area detector computed tomography and evaluation of its clinical usefulness. Neurosurgery, 69(4):843 $851,2011$.

[13] Y. Hoi, H. Meng, S. Woodward, B. Bendok, R. Hanel, L. Guterman, and L. Hopkins. Effects of arterial geometry on aneurysm growth: Three-dimensional computational fluid dynamics study. Journal of Neurosurgery., 101:676 - 681, 2004.

[14] T. Horikoshi, M. Sugita, T. Yagishita, and H. Nukui. Size of cerebral aneurysms and related factors in patients with subarachnoid hemorrhage. Surgical Neurology, 61:239 $-245,2002$.

[15] F. Jourdan and A. Samida. An implicit numerical method for wear modeling applied to hip joint prothesis problem. Computer Methods in Applied Mechanics and Engineering, 198(27-29):2209-2217, 2009. 
[16] C. Karmonik, O. Diaz, R. Grossman, and R. Klucznik. In vivo quantification of wall motion in cerebral aneurysm from $2 \mathrm{D}$ cine phase contrast magnetic resonance images. Rofo, 182(2):140-151, 2010.

[17] E. Malachanne, D. Dureisseix, and F. Jourdan. Numerical model of bone remodeling sensitive to loading frequency through a poroelastic behavior and internal fluid movements. Journal of the Mechanical Behavior of Biomedical Materials, 4(6):849-857, 2011.

[18] S. Matsubara, H. Hadeishir, A. Suzukil, N. Yasui, and B. Nishimura. Incidence and risk factors for the growth of unruptured cerebral aneurysms: observation using serial computerized tomography angiography. Journal of Neurosurgery, 101(6):908 - 914, 2004.

[19] P. Moireau, N. Xiao, M. Astorino, C. Figueroa, D. Chapelle, C. Taylor, and J. Gerbeau. External tissue support and fluid - structure simulation in blood flows. American Journal of Neuroradiology, 1617-7959(10.1007/s10237-011-0289-z):1-18, 2011.

[20] A. Molyneux. International subarachnoid aneurysm trial (ISAT) of neurosurgical clipping versus endovascular coiling in 2143 patients with ruptured intracranial aneurysms: a randomised trial. The Lancet, 360(9342):1267-1274, 2002.

[21] B. Nahed, M. Diluna, T. Morgan, E. Ocal, A. Hawkins, K. Ozduman, K. Kahle, A. Chamberlain, and A. Amar. Hypertension, age, and location predict rupture of small intracranial aneurysms. Neurosurgery, 57:676 - 683, 2005.

[22] F. Nicoud, R. Moreno, B. Tayllamin, M. Chau, and H. Rousseau. Computational hemodynamics in moving geometries without solving the fluid-structure interaction problem. In Conference on Modelling Fluid Flow 2009. The 14th International Conference on Fluid Flow Technologies. Budapest, Hungary, September 9-12, 2009, 2009. 
[23] J.K. Park, C. Lee, K. Sim, J. Huh, and J.C. Park. Imaging of the walls of saccular cerebral aneurysms with double inversion recovery black-blood sequence. Journal of Magnetic Resonance Imaging, 30(5):1179 - 1183, 2009.

[24] J. Penrose and C. Staples. Implicit fluid-structure coupling for simulation of cardiovascular problems. International Journal for Numerical Methods in Fluids, 40(3-4):467 $-478,2002$.

[25] P. Rissland, Y. Alemu, S. Einav, J. Ricotta, and D. Bluestein. Abdominal aortic aneurysm risk of rupture: Patient-specific FSI simulations using anisotropic model. Journal of Biomechanical Engineering, 131(3):031001, 2009.

[26] D. Sforza, C Putman, and J. Cebral. Hemodynamics of cerebral aneurysms. The Annual Review of Fluid Mechanics, 41:91 - 107, 2008.

[27] L. Speelman, E. Bosboom, G. Schurink, J. Buth, M. Breeuwer, M. Jacobs, and F. van de Vosse. Initial stress and nonlinear material behavior in patient-specific AAA wall stress analysis. Journal of Biomechanics, 42(11):1713 - 1719, 2009.

[28] L. Steiner and J. Andrews. Monitoring the injured brain : Icp and cbf. British Journal of Anaesthesia, 97(1):26 - 38, 2006.

[29] P. Swider, A. Pédrono, D. Ambard, F. Accadbled, and J. Sales de Gauzy. Substructuring and poroelastic modelling of the intervertebral disc. Journal of Biomechanics, 47(7):1287-1291, 2010.

[30] R. Torii, M. Oshima, T. Kobayashi, K. Takagi, and T. Tezduyar. Fluid structure interaction modeling of a patient-specific cerebral aneurysm: influence of structural modeling. Computational Mechanics, 43(1):151 -159, 2008. 
[31] A. Valencia, D. Ledermann, R. Rivera, E. Bravo, and M. Galvez. Blood flow dynamics and fluid-structure interaction in patient specific bifurcating cerebral aneurysm. International Journal for numerical methods in fluids, 58:1081 - 1100, 2009.

[32] A. Valencia and F. Solis. Blood flow dynamics and arterial wall interaction in a saccular aneurysm model of the basilar artery. Computers and Structures, 84:1326 1337, 2006.

[33] J. Vande Geest, M. Sacks, and D. Vorp. The effects of aneurysm on the biaxial mechanical behavior of human abdominal aorta. Journal of Biomechanics, 39(7):1324 $-1334,2006$.

[34] J. Vande Geest, E. Schmidt, M. Sacks, and D. Vorp. The effects of anisotropy on the stress analyses of patient-specific abdominal aortic aneurysms. Annals of Biomedical Engineering, 36(6):921 - 932, 2008.

[35] R. Vito and J. Hickey. The mechanical-properties of soft-tissues. 2. The elastic response of arterial segments. Journal of Biomechanics, 13(11):951 -957, 1980.

[36] J. Wardlaw and P. White. The detection and management of unruptured intracranial aneurysms. Brain, 123(2):205 -221, 2000.

[37] P. Watton, N. Raberger, G. Holzapfel, and Y. Ventikos. Blood flow dynamics and fluid-structure interaction in patient specific bifurcating cerebral aneurysm. Journal of Biomechanical Engineering, 131(101003):1 - 14, 2009.

[38] N. Yang and K. Vafai. Modeling of low-density lipoprotein (LDL) transport in the artery- effects of hypertension. International Journal of Heat and Mass Transfer, $49: 850-867,2006$.

[39] P. Young, T. Beresford-West, S. Coward, B. Notarberardino, B. Walker, and A. AbdulAziz. An efficient approach to converting 3D image data into highly accurate com- 
putational models. Philosophical Transactions of the Royal Society A, (366):3155 $3173,2008$.

[40] C. Zhang, M. Villa-Uriol, M. Craene, J. Pozo, and A. Frangi. Morphodynamic analysis of cerebral aneurysm pulsation from time-resolved rotational angiography. Medical Imaging, 28(7):1105-1116, 2009. 


\section{$6 \quad$ Figures}

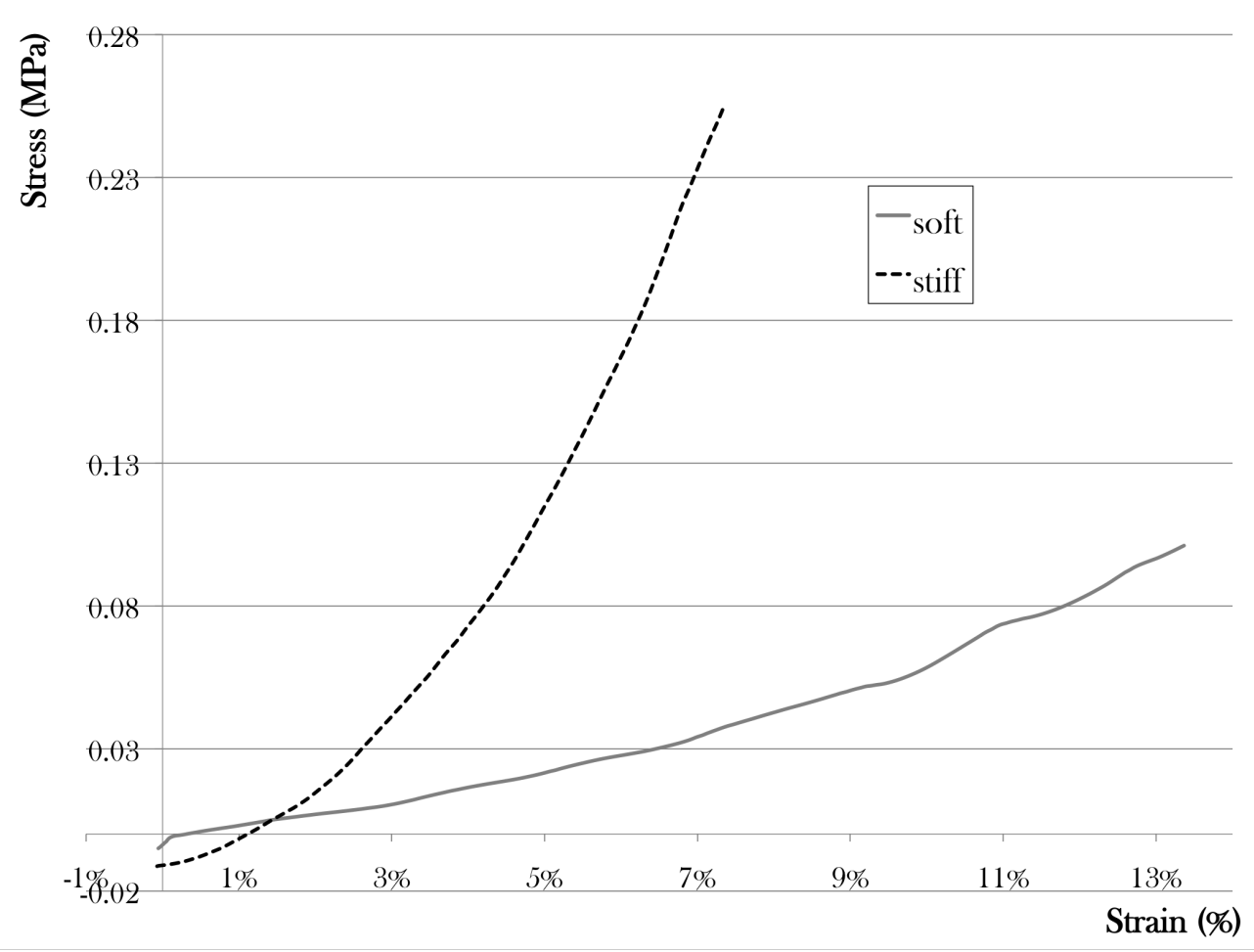

Figure 1: Nominal stress/engineering strain curves representing the average mechanical properties of the soft and the stiff classes. 


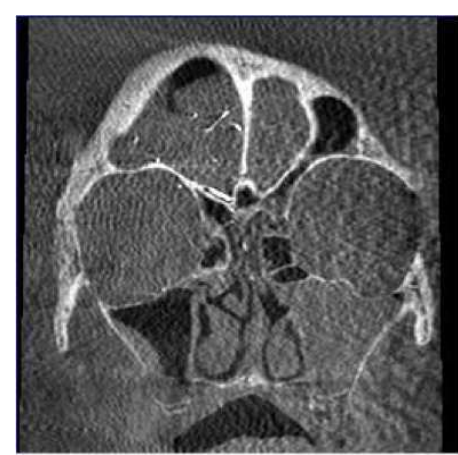

(a)

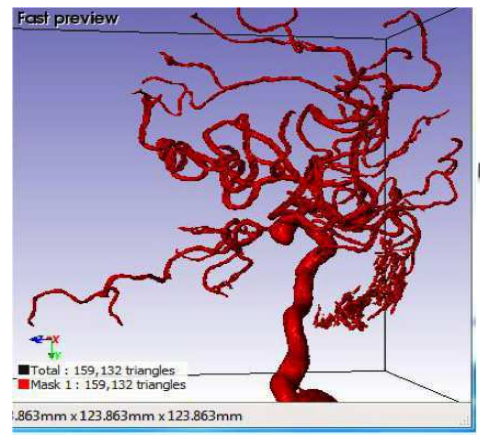

(c)

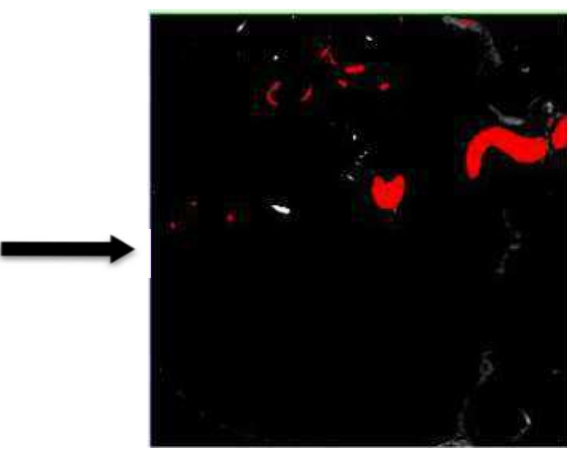

(b)

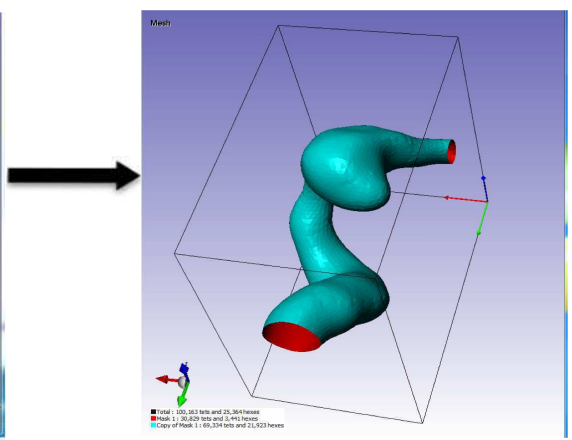

(d)

Figure 2: Modelling of a 3D geometry from 2D images (dicoms). (a) Dicom image from medical exam; (b) threshold step: selection of the voxels in the image having a grey level value inside a user-prescribeb range (the first segmentation step) ; (c) 3D view before segmentation; (d) 3D object after segmentation and smoothing 
Figure 3: Imposed volumetric flow rate and pressure at the inlet and outlet of the computational domain (artery).

On the pressure curve : Point A corresponds to the diastolic state, Point B corresponds to the systolic state and the line $\mathrm{C}$ represents the average of geometry acquisition. 
Figure 4: Display of the structural mesh (top), 3110 elements, $\Omega^{s}$ with the border $\partial \Omega^{s}$ and of the fluid mesh, 102,562 elements (bottom). 


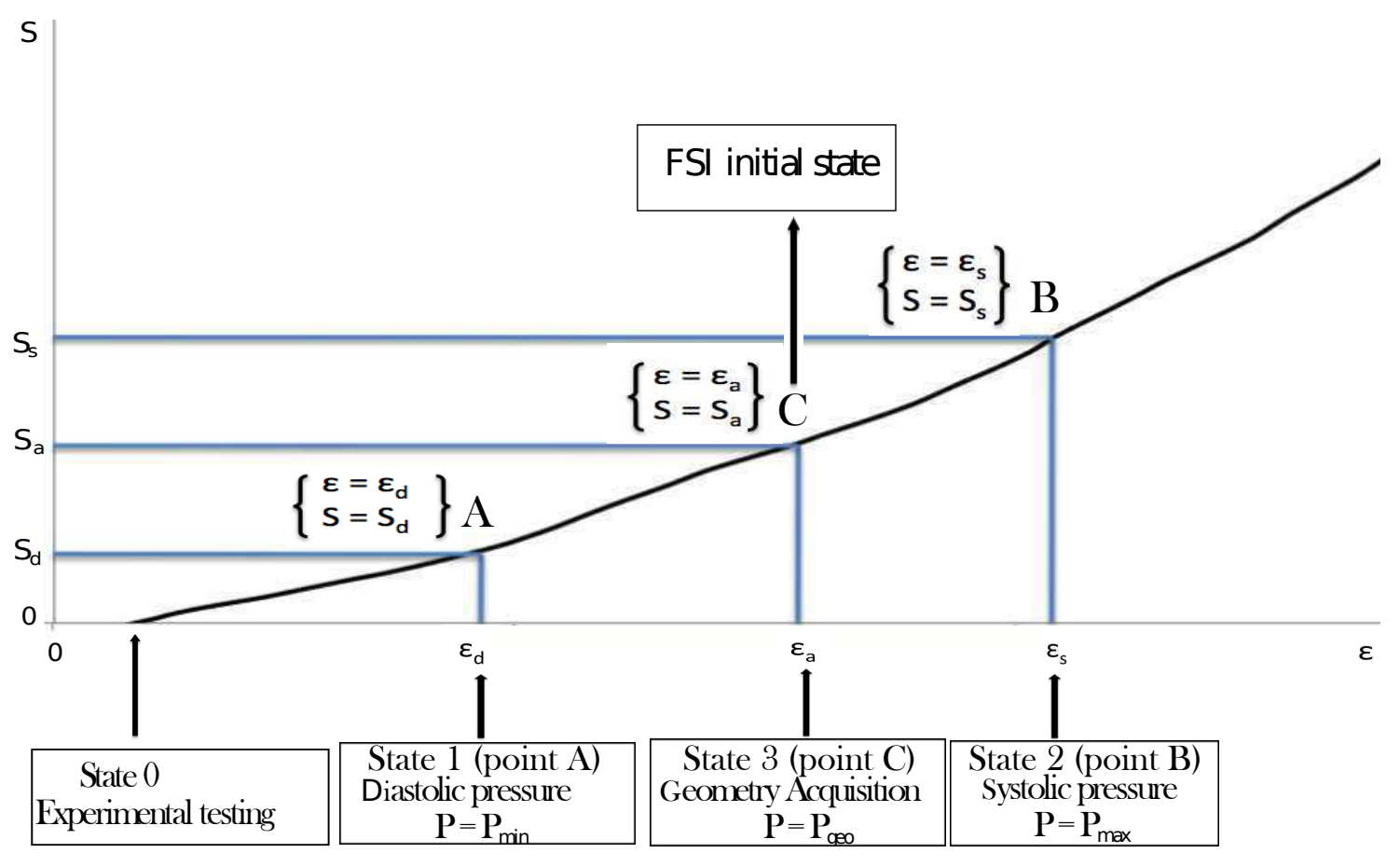

Figure 5: Definition of the four states of the tissue 
7

8
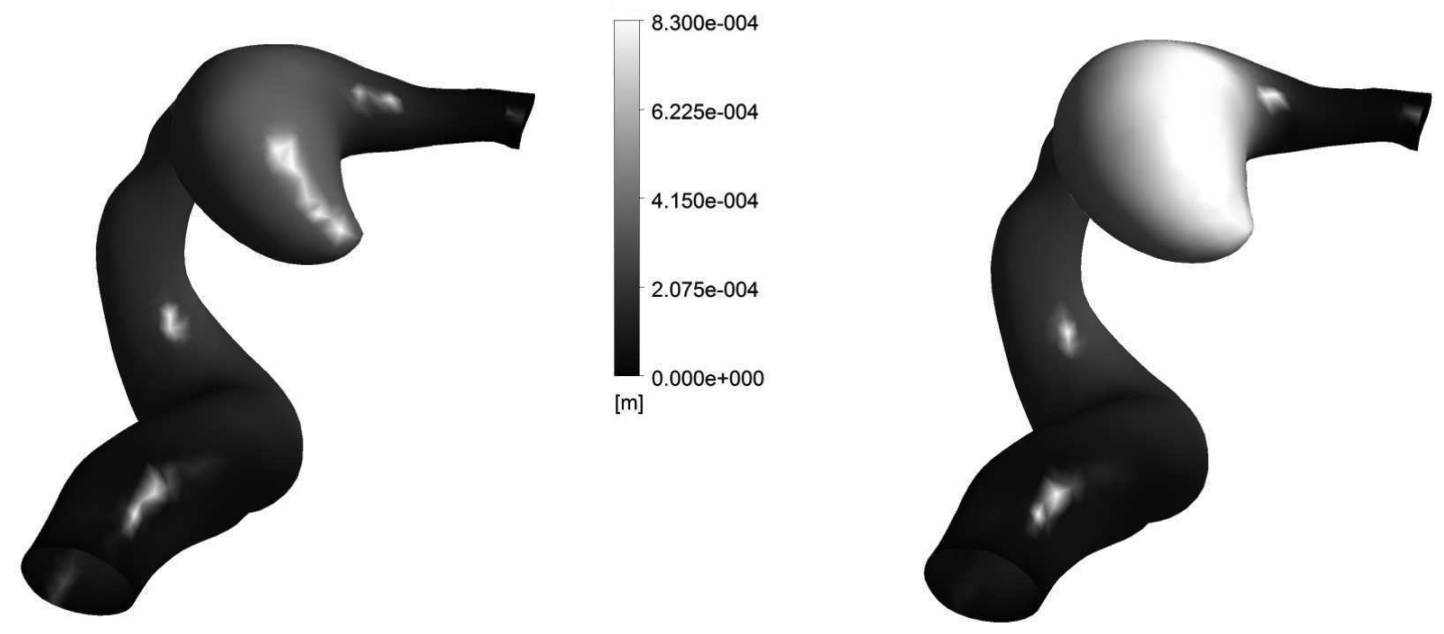

Figure 6: Total mesh displacement with initial stress at the systole: for both the stiff aneurysm (left) and the soft aneurysm (right). 


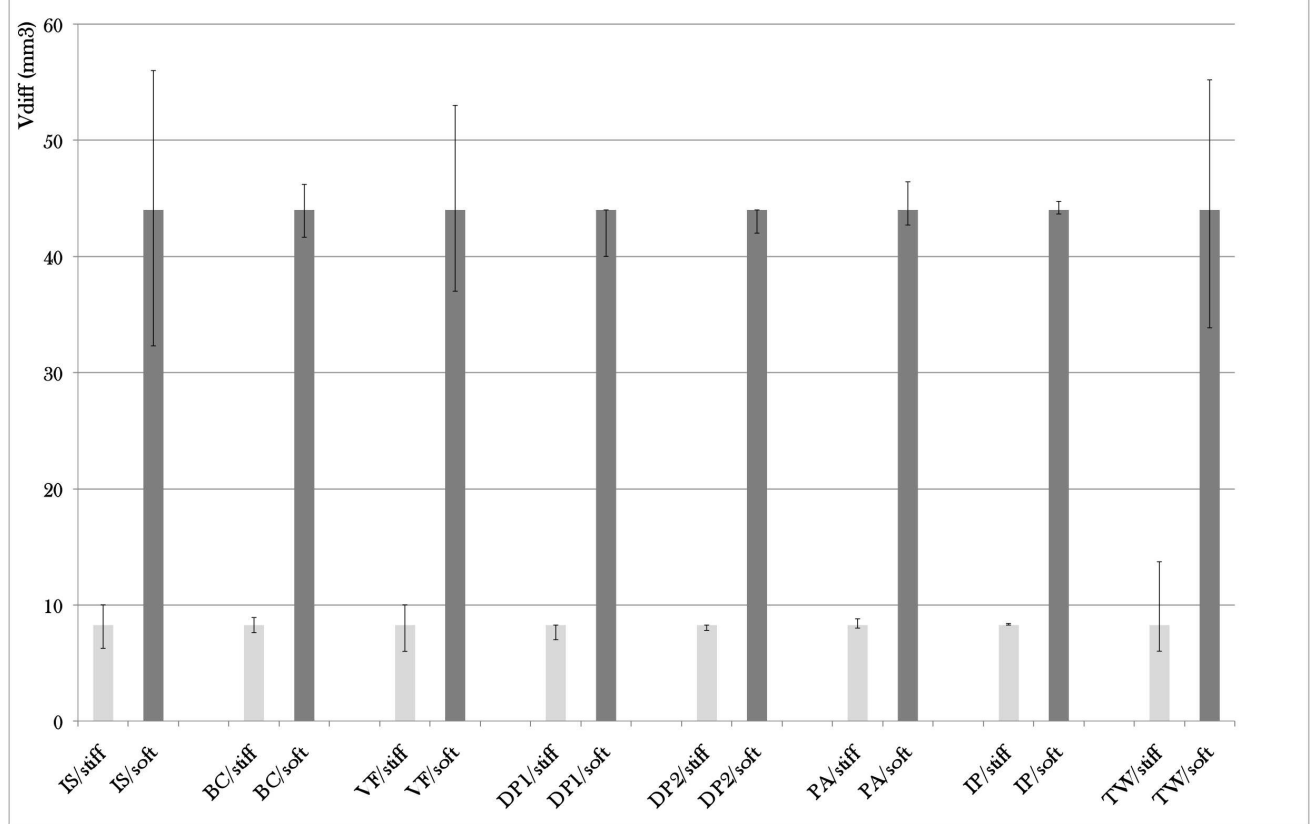

Figure 7: Results of the influence of the parameters tested in the parametric study. Where IS $=$ Initial Stress, $\mathrm{BC}=$ Flow Boundary Conditions, VF $=$ Volumetric Flow Rate, DP1 $=\triangle \mathrm{P}$ of $35 \mathrm{mmHg}, \mathrm{DP} 2=\triangle \mathrm{P}$ of $45 \mathrm{mmHg}, \mathrm{PA}=$ Parent Artery, $\mathrm{IP}=$ Intracranial Pressure and TW $=$ Thickness of the aneurysm Wall. 


\section{Tables}

\begin{tabular}{|c|c|c|c|}
\hline Aneurysm & $C_{10}(\mathrm{MPa})$ & $C_{01}(\mathrm{MPa})$ & $C_{11}(\mathrm{MPa})$ \\
\hline stiff & 0.39 & 0 & 22.14 \\
\hline soft & 0.024 & 0.026 & 0.42 \\
\hline
\end{tabular}

Table 1: Material coefficients of the aneurysm walls used in the present study. 


\begin{tabular}{|c|c|c|}
\hline Aneurysm & $\mathrm{D}_{\max }(\mathrm{mm})$ & $\mathrm{V}_{\text {diff }}\left(\mathrm{mm}^{3}\right)$ \\
\hline stiff & 0.37 & 8.25 \\
\hline soft & 0.77 & 44 \\
\hline
\end{tabular}

Table 2: Comparaison between the results of FSI computations for the two different aneurysmal tissues (stiff and soft) 


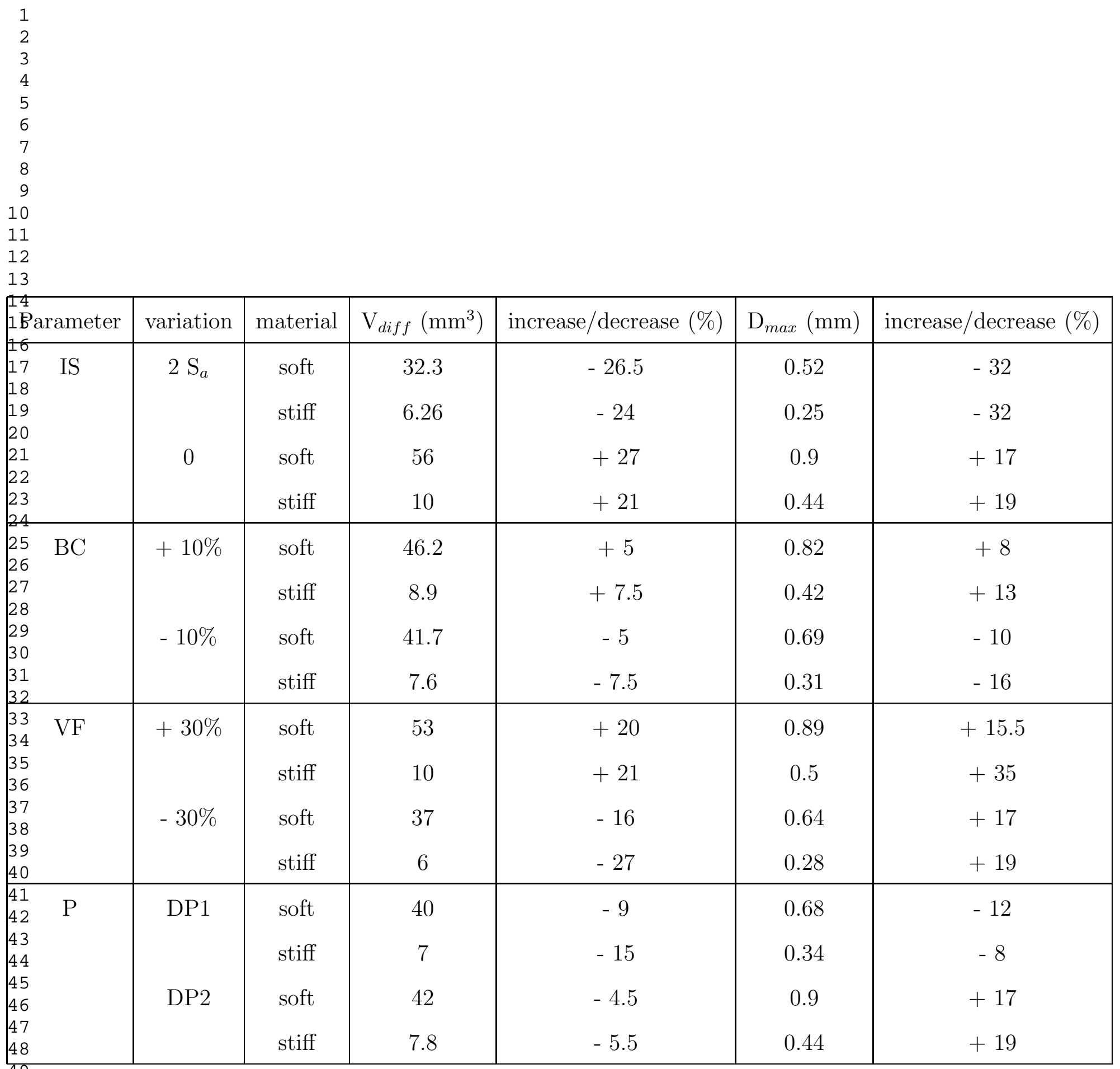




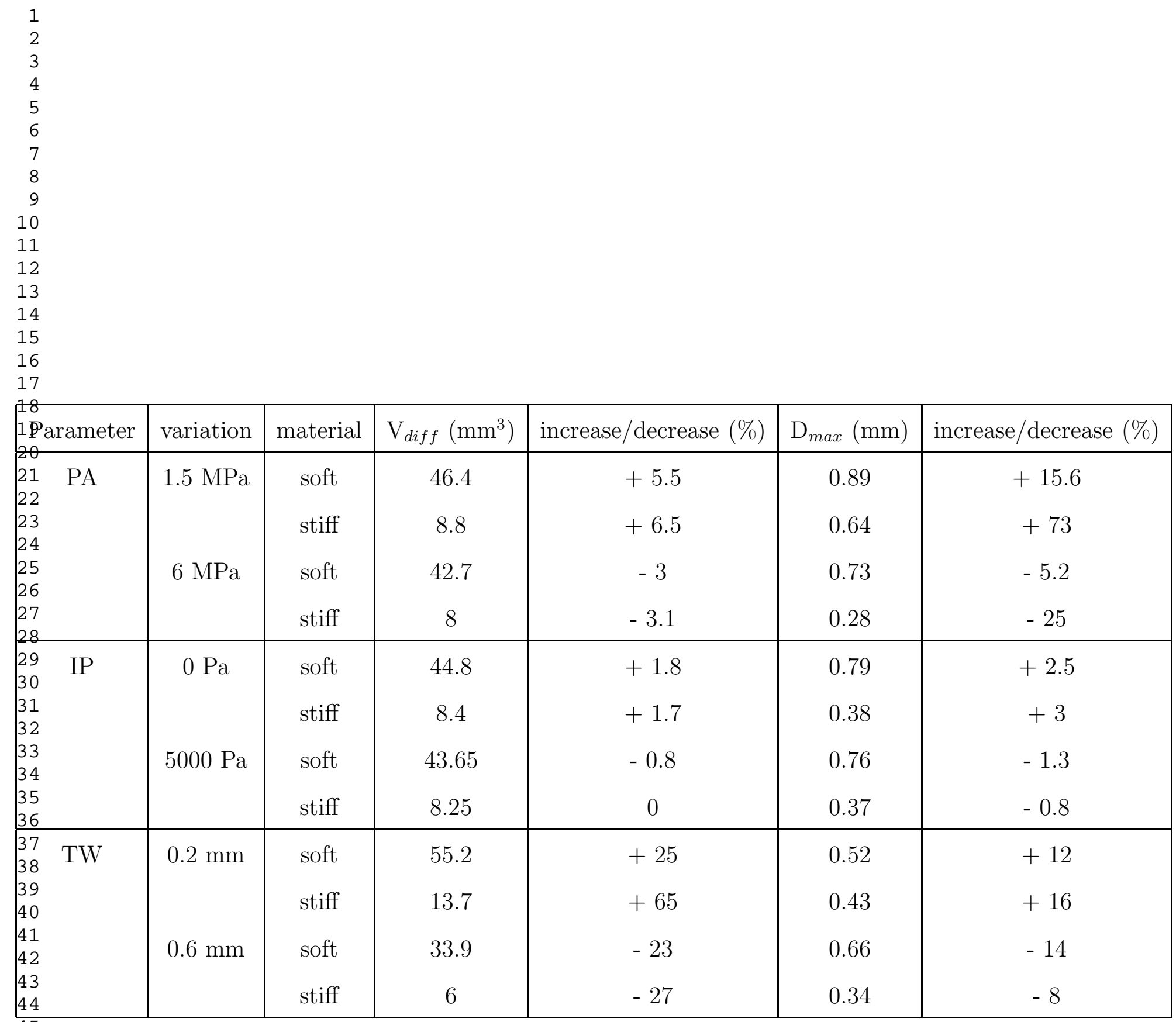

Table 4: Results of the parametric study.
Where PA $=$ Parent Artery, IP $=$ Intracranial Pressure and TW $=$ Thickness of the aneurysm Wall. 